\title{
Oil saturation distribution in polluted aquifer result from quadrupole wells flow field configuration
}

\author{
Arieh Pistiner ${ }^{1}$ \\ (C) Springer Nature Switzerland AG 2019
}

\begin{abstract}
A new physico-mathematical approach is developed for modeling oil saturation distribution within a plain polluted aquifer subjected to a flow field governed by a quadrupole wells system. This system consists of two extraction and two injection (2E/2I) wells, located in a contaminated aquifer, which are used for groundwater remediation applications. The development of the model is done by considering a two-dimensional, nonlinear diffusion-convection transport equation, applying a geometrical self-similar scheme. A closed-form analytical solution is obtained for the steady oil saturation distribution in the aquifer for the above-mentioned flow field. The mathematical solution developed here is designed to simulate hydraulic interactions of $2 \mathrm{E} / 2 \mathrm{I}$ wells with the oil polluted aquifer.
\end{abstract}

Keywords Aquifer - Oil saturation - Quadrupole wells configuration · Two-dimensional saturation distribution . Geometrical self-similar solution

\section{Introduction}

Remediation of contaminated groundwater may involve the injection of water into the aquifer to stimulate degradation of oil contaminants, coupled with the removal of water from extraction wells to increase circulation. Ideally, the water should be mixed throughout the oil contaminated area to optimize the reaction rate. However, due to the laminar flows characteristic in porous media, mixing in groundwater is an inherently slow process governed by molecular diffusion. In contrast, mixing occurs more readily in rotational flows, such as the turbulent flow in streams [9] using groundwater circulation well (GCW) systems technology.

Subsurface double-well pumping serves to impede the groundwater flow and is often used in conjunction with groundwater extraction wells, as components of pump and treat systems. Such wells are also used to contain contaminated groundwater and in groundwater treatment systems. They are frequently used with surface caps to produce an essentially complete containment structure.
Anderson and Mesa [2] presented an analytic solution describing the discharge vector for a two-dimensional, steady groundwater flow past an impermeable barrier, embedded in a regional flow field. In their study, the hydraulic containment of contaminant plumes was examined by combining a vertical barrier and extraction wells. A closed-form self-similar solution is also obtained by Pistiner and Shapiro [13] and Pistiner [11] which describes the saturation distribution within a two-dimensional isotropic and anisotropic aquifer of a pollutant discharging from an underground source. Later, Pistiner [12] proposed a self-similar solution for a two-dimensional steady-state oil plume distribution in an aquifer near an impermeable barrier. Satkin and Bedient [16] evaluated the effectiveness of seven different well patterns for restoring contaminated groundwater under eight generic hydrogeologic conditions. It was found that the doublet configuration is to minimize cleanup time, the volume of water circulated, and the volume of water treated. Antonacci et al. [3] specified several criteria for case studies of groundwater contamination events, analyzed by large-scale numerical

Arieh Pistiner, ariehpistiner@gmail.com | 1 Ministry of the Environmental Protection, 15 a Pal-Yam St., Haifa 33095, Israel. 
models, results from sink and/or source conditions, which have a major impact on the concentration distribution in the aquifer. Zeidan [17] described in here study groundwater pollution and remediation schemes in the Nile Delta, together with numerical study which involve the use of injections and extractions wells. Bear and Cheng [5] presented methodology and procedure for constructing complete conceptual and mathematical models of groundwater contaminant transport by using pumping and injection wells. These models are used as essential tools for the planning and management for sustainable use of groundwater resources. The use of the mathematical models is based on physical assumptions, subjected to acceptable approximations and limitations.

In this study, we present a closed-form analytic solution describing the effects of $2 \mathrm{E} / 2 \mathrm{I}$ wells, lying in a quadrupole scheme, on the distribution of an oil contamination plume in the polluted aquifer. The steady-state distribution of oil contaminants in a saturated aquifer is analyzed and simulated by applying a geometrical self-similar model. The model employs the basic equations of the two-phase flow. In addition we assume some power law relationship which is supposed to exist between oil saturation, capillary pressure and relative permeability. The self-similar model pertains to cases wherein both space coordinates in the oil transport equations can be replaced by a single variable. This solution is relevant in a preliminary design of remedial systems and in particular, we examine the plume dimension and the oil flux near the four wells in order to control the oil plume distribution in the aquifer. This calculation yields valuable similarity solutions. On this basis, the model is calibrated (e.g., without using data such as fluid densities and viscosities, soil permeability, etc.), by matching hypothetical saturation field data, taken from several observation wells and is further used to calculate the actual saturation in every location in the aquifer.

It should be noted that analytical solutions are usually solved under certain assumptions (e.g. aquifer homogeneity, neglect of gravity, saturation profiles of a certain shape) which make them appear less applicable to real world problems. However, analytical solutions have a value of their own, simply by allowing a quick assessment of what roughly to expect and, more importantly, can be used to validate implementations of numerical models.

\section{Physico-mathematical model}

Consider a two-dimensional homogeneous and isotropic porous layer (e.g., an aquifer), on a regional scale, that is, over a planar horizontal extent much larger than its thickness. Consider a case where a large portion of the aquifer is saturated with oil contaminant, i.e. the irreducible water saturation is 0.5 while the oil saturation in the contaminated aquifer reached the maximal possible value, i.e. $S \approx 0.5$ [8]. This zone will be defined in this study as the "free layer" zone, which is characterized by connected oil blobs [10]. The remediation system of this zone contains two pairs of extraction and injection wells. As such, water is extracted from deep strata in the contaminated aquifer via two wells. The extracted water is re-injected into the aquifer through a pair of injection wells, located very close to the extraction wells. As a result, oil is displaced toward the extraction wells by the water and the oil saturation near the injection wells is reduced and decreases down to the value $S=0.5$. This zone, where the oil saturation is smaller than $0.5(S<0.5)$ will be defined in this study as the transition zone, which is characterized by large amount of disconnected oil blobs. Although the capillary forces that hold residual oil in pores are relatively strong, they can be overcome to some degree by a sweep scenario involved injecting water on one side of the aquifer and extracting of from the other side. As a result, the water will sweep (removing) oil contamination from those zones which are closed to the wells.

The respective specific discharge vectors $\mathrm{q}_{w}$ and $\mathrm{q}_{o}$ of the water and the oil in the transition zone in the absence of the gravity force, are modeled by Darcy's law. We can write this for each fluid in a Cartesian system, using the double index summation convention $x_{1}=x$ (and $x_{2}=x$ ) in the form

$q_{w_{i}}=-\kappa_{i j} \frac{K_{r w}(S)}{\mu_{w}} \frac{\partial P_{w}}{\partial x_{j}}, \quad i=1,2$

and

$q_{o_{i}}=-\kappa_{i j} \frac{K_{r o}(S)}{\mu_{o}} \frac{\partial P_{o}}{\partial x_{j}}, \quad i=1,2$

where $S$ is the macroscopic (averaged over the layer height) oil saturation in the porous medium $(0<S<1)$ (i.e., the water saturation equal to $1-S) ; K_{r w}(S), K_{r o}(S)$ are the water and oil relative permeabilities, respectively. Other quantities appearing in (1a) and (1b) are: $\kappa_{i j}$-the Cartesian components of the permeability tensor, $\mu_{o}, \mu_{w}$-the oil and water viscosities, respectively, $P_{o}$ and $P_{w}$ - the oil and water pressures respectively, which are related via the capillary pressure $P_{c}$

$P_{c}(S)=P_{o}-P_{w}$.

The conservation of mass for each fluid in a rigid porous medium of porosity $\phi$ yields

$\frac{\partial q_{w_{i}}}{\partial x_{i}}-\phi \frac{\partial S}{\partial t}=0, \quad i=1,2$ 
$\frac{\partial q_{o_{i}}}{\partial x_{i}}+\phi \frac{\partial S}{\partial t}=0, \quad i=1,2$

where $t$ is the time variable. The pair Eqs. (3a) and (3b) automatically gives the mass balance equation

$\frac{\partial q_{i}}{\partial x_{i}}=0, \quad i=1,2$,

where

$q_{i}=q_{w_{i}}+q_{o_{i}} \cdot \quad i=1,2$,

and the steady flow in the aquifer is subjected to the flow field created by pair of extraction and injection (2E/2I) wells, located very close to each other.

Combining (1a), (1b)-(5) we obtain

$\phi \frac{\partial S}{\partial t}=\frac{\partial}{\partial x_{i}}\left(\psi(S) \kappa_{i j} \frac{\partial S}{\partial x_{j}}-q_{i} F(S)\right), \quad i=1,2$

where the convection term $F(S)$ and the capillary dispersion term $\psi(S)$ are given by

$$
\begin{aligned}
& F(S)=\left(1+\frac{K_{r w}(S) \mu_{o}}{K_{r o}(S) \mu_{w}}\right)^{-1}, \\
& \psi(S)=\frac{K_{r w}(S)}{\mu_{w}} \frac{\mathrm{d} P_{c}(S)}{\mathrm{d} S} F(S) .
\end{aligned}
$$

Since the slope $\mathrm{d} P_{c}(S) / \mathrm{d} S \rightarrow \infty$ as the oil saturation approaches zero, we may assume [15] that

$\frac{\mathrm{d} P_{c}(S)}{\mathrm{d} S} \sim S^{-m} \quad S \rightarrow 0$,

where the parameters $m$ in (8) were found to depend on the porous medium properties [14].

We will further assume that the aquifer is homorganic and isotropic (i.e., $\kappa_{x y}=\kappa_{y x}=0$ and $\kappa_{x x}=\kappa_{y y}=\kappa$ ) and that the value of $S$ is small, especially near the wells where the sweep up of residual oil supposed to be very effective. Accordingly, the fractional flow curve $F(S)$ and the function $\psi(S)$ can be represented by power-law dependence [14], usually valid for low oil saturation

$F(S) \approx F_{0} S^{n}$

$\psi(S) \approx \frac{\gamma_{o w}}{\mu_{w}} \psi_{0} S^{n-m}$.

Here $\gamma_{o w}$ is the oil-water surface tension, $F_{0}$ is a dimensionless factor of order one, and $\psi_{0}$ is of the order of $\tilde{P}_{c} / \gamma_{o w}$ where $\tilde{P}_{c}$ is the characteristic capillary pressure in the aquifer. Parameters $m$ and $n$ were found to depend on the pore-size index of the porous medium and the oil viscosities [14]. From the experimental data on fractional flow rates and permeabilities it was found that $m$ and $n$ typically vary in the following ranges: $1<n<3$, $1.5<m<4$. It should be noted that $(9 \mathrm{~b})$ assumes negligible changes in $K_{r w}(S)$ (appears in (7b)) for low oil saturation.

We assume that initially at $\hat{t}=-0$ the aquifer is contaminate with free layer in very large portion of the aquifer such that

$S(x, y)=0.5, \quad-\infty<x<+\infty, \quad-\infty<y<+\infty$.

We assume that a steady-state oil saturation distribution is established a sufficiently long time (i.e., $t \rightarrow \infty$ ) after the beginning of the Extractions/Injections process [13]. In such a case, the term on the left-hand side of (6) vanishes (i.e., $\partial S / \partial t \rightarrow 0$ ) and Eq. (6) describe a steady-state distribution on the oil saturation $S(x, y)$ within the porous medium. Both terms (c.f., Eq. (6)) of capillary dispersion and oil convection, in the $x$ and the $y$ directions, manifests themselves in such a way that the distribution of the oil saturation in the aquifer may exists under a steady-state condition. This condition exists as long as the $2 \mathrm{E} / 2 \mathrm{l}$ process is going on and the aquifer area, far from both wells, remains in a "free layer" state and behaves as an infinite source of oil to the transition zone. Despite the fact that the model is more appropriate to a case where the oil saturation is not high, we will assume that the iso-saturation line $S=0.5$ may serve as the boundary between the "free layer" zone and the transition zone (e.g., $0<S<0.5$ ). This iso-saturation line will be obtained as a part of the solution.

We will assume that the flow in the aquifer is much slower compared with the flow created by the two extraction and two injection wells in a quadrupole configuration. In addition to this we assume that the distance between all wells is very small. Under the above assumptions, the streamline function $\Psi(x, y)$ (shown in Fig. 1 ) and the specific discharge components, resulting from a quadrupole flow field are given by [6]

$\Psi(x, y)=-Q \frac{2 x y}{\left(x^{2}+y^{2}\right)^{2}}$

$q_{x}=\frac{\partial \Psi}{\partial y}=Q \frac{-2 x^{3}+6 x y^{2}}{\left(x^{2}+y^{2}\right)^{3}}$

$q_{y}=-\frac{\partial \Psi}{\partial x}=Q \frac{2 y^{3}-6 y x^{2}}{\left(x^{2}+y^{2}\right)^{3}}$

where $Q$ is the discharge intensity of the water (c.f. in volume per time) into and from the wells. The fluxes represented in (11a) and (11b), are created by stream lines that 
Fig. 1 Stream lines paths in aquifer results from 2 injection and 2 extraction wells in quadrupole configuration. Source: https://he.wikipedia.org/wiki/ \%D7\%A7\%D7\%95\%D7\%91\%D 7\%A5:VFPt_quadrupole_xy.svg

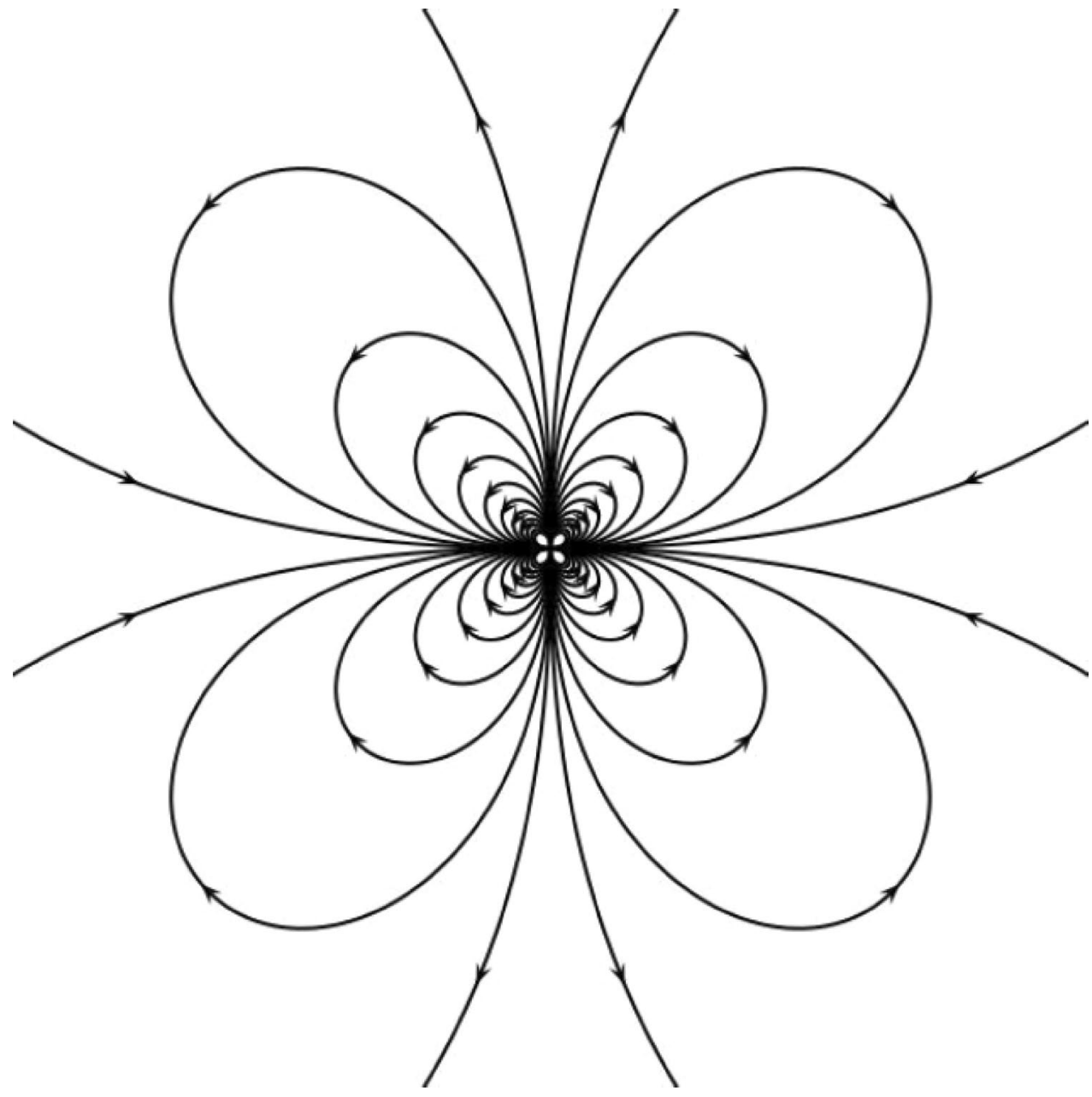

connect two extraction wells lying on the $x$ axis (at $x=+0$ and $x=-0$ ) and two injection wells lying on the $y$ axis (at $y=+0$ and $y=-0$ ). As such, the regions near the $y$ axis will be defined in this study as the upstream zone (positive and negative directions with respect to $y=0$ ) and the regions near the $x$ axis will be defined in this study as the downstream zone (positive and negative directions with respect to $x=0$ ).

We will define now a modified and saturation, as follows: $\tilde{S} \equiv S^{n-m+1}$.

We will also defined the dimensionless variables $\hat{x}$ and $\hat{y}$, related to their Cartesian counterparts as follows:

$\hat{x}=\frac{x}{L}$,

$\hat{y}=\frac{y}{L^{\prime}}$

where $L$ is a characteristic length of the aquifer which can be obtained by the introduction of (12), (13a) and (13b) into (6), (9a), (9b), (11a) and (11b) as follows:

$$
\begin{aligned}
0= & \frac{1}{L} \frac{\partial}{\partial \hat{x}}\left(\frac{\kappa \gamma_{o w} \Psi_{o}}{L \mu_{w}(n-m+1)} \frac{\partial \tilde{S}}{\partial \hat{x}}-\frac{F_{o} Q}{L^{2}} \tilde{S} \frac{n}{1+n-m} \frac{\hat{y}^{2}-\hat{x}^{2}}{\left(\hat{x}^{2}+\hat{y}^{2}\right)^{2}}\right) \\
& +\ldots \frac{1}{L} \frac{\partial}{\partial \hat{y}}\left(\frac{\kappa \gamma_{o w} \Psi_{o}}{L \mu_{w}(n-m+1)} \frac{\partial \tilde{S}}{\partial \hat{y}}+\frac{F_{o} Q}{L^{2}} \tilde{S}^{\frac{n}{1+n-m}} \frac{2 \hat{x} \hat{y}}{\left(\hat{x}^{2}+\hat{y}^{2}\right)^{2}}\right) .
\end{aligned}
$$

Multiplying both sides of (14a) with the term $\frac{L^{3}}{Q F_{o}}$ we obtain

$\begin{aligned} 0= & \frac{\partial}{\partial \hat{x}}\left(\frac{\hat{y}^{2}-\hat{x}^{2}}{\left(\hat{x}^{2}+\hat{y}^{2}\right)^{2}} \tilde{S}^{\frac{n}{1+n-m}}-\frac{L \kappa \gamma_{o w} \Psi_{o}}{\mu_{w} Q F_{o}(n-m+1)} \frac{\partial \tilde{S}}{\partial \hat{x}}\right) \\ & +\ldots \frac{\partial}{\partial \hat{y}}\left(-\frac{2 \hat{x} \hat{y}}{\left(\hat{x}^{2}+\hat{y}^{2}\right)^{2}} \tilde{S}^{\frac{n}{1+n-m}}-\frac{L \kappa \gamma_{o w} \Psi_{o}}{\mu_{w} Q F_{o}(n-m+1)} \frac{\partial \tilde{S}}{\partial \hat{y}}\right) .\end{aligned}$

By equalizing the term $\frac{L \kappa \gamma_{o w} \Psi_{o}}{\mu_{\mathrm{w}} \mathrm{Q} F_{o}(n-m+1)}$ to 1 , we obtain the characteristic length of the aquifer

$L=\frac{Q \mu_{w} F_{o}(n-m+1)}{\gamma_{o w} \Psi_{o} \kappa}$, 
where the product of $F_{o}(n-m+1)$ appearing in (14c) is a positive dimensionless factor [11].

In dimensionless form, Eq. (14b) subjected to (14c) can be rewritten as follows:

$\frac{\partial J_{\hat{x}}}{\partial \hat{x}}+\frac{\partial J_{\hat{y}}}{\partial \hat{y}}=0$,

where

$J_{\hat{x}}=\frac{-2 \hat{x}^{3}+6 \hat{x} \hat{y}^{2}}{\left(\hat{x}^{2}+\hat{y}^{2}\right)^{3}} \tilde{S}^{\frac{n}{1+n-m}}-\frac{\partial \tilde{S}}{\partial \hat{x}}$

$J_{\hat{y}}=\frac{2 \hat{y}^{3}-6 \hat{y} \hat{x}^{2}}{\left(\hat{x}^{2}+\hat{y}^{2}\right)^{3}} \tilde{S} \frac{n}{1+n-m}-\frac{\partial \tilde{S}}{\partial \hat{y}}$.

In the next section we will develop a similarity solution, which is valid in the transition zone at the range of $0<\tilde{S}(\hat{x}, \hat{y}) \leq 0.5$.

\section{Self-similar model}

We now refer to the circumstances in which the steadystate oil distribution, described by the nonlinear PDE (15), (15a) and (15b), achieves a certain asymptotic, derived from universal behavior and described by a single independent variable $\xi=\hat{x} / \hat{y}[4]$

$\tilde{S}(\hat{x}, \hat{y})=f(\xi) \hat{y}^{2}$,

$\xi=\hat{x} / \hat{y}$,

where $f(\zeta)$ is a similarity function and is valid for the particular case

$n=2(m-1)$.

The specific discharge components, resulting from a flow field as described in Fig. 1, are obtained after the introduction of (16b) into (11a) and (11b), using (13a) and (13b) as follows

$q_{x}=\hat{y}^{-3} U(\xi)$

$q_{y}=\hat{y}^{-3} V(\xi)$,

where

$U(\xi)=\frac{-2 \xi^{3}+6 \xi}{\left(1+\xi^{2}\right)^{3}}$

$V(\xi)=\frac{2-6 \xi^{2}}{\left(1+\xi^{2}\right)^{3}}$.
Introducing (16a), (16b), (16c), (17a), (17b), (18a) and (18b) into (15), (15a) and (15b) we obtain

$J_{\hat{x}}=\hat{y} g(\xi)$,

$J_{\hat{y}}=\hat{y} h(\xi)$,

where

$g(\xi)=U f^{2}-f^{\prime}$,

and

$h(\xi)=V f^{2}-2 f+f^{\prime} \xi$,

where $g(\xi)$ and $h(\xi)$ are the flux components and $f^{\prime} \equiv \frac{\mathrm{d} f}{\mathrm{~d} \xi}$. Using (16b), (19a) and (19b) we obtain the following expressions

$\frac{\partial J_{\hat{x}}}{\partial \hat{x}}=g^{\prime}$,

and

$\frac{\partial J_{\hat{y}}}{\partial \hat{y}}=2 h-(h \xi)^{\prime}$.

Substituting (21a) and (21b) in (15) we obtain

$(g-h \xi)^{\prime}+2 h=0$.

Introducing (20b) into (22) we obtain

$\frac{\mathrm{d}(g-h \xi+2 f \xi)}{\mathrm{d} \xi}=-2 V f^{2}+6 f$.

By using (23), we define now a new variable $w(\xi)$ as follows

$w=g-h \xi+2 f \xi$.

Introducing (20a) and (20b) into (24) and using (23) we obtain the pair of equations

$w=f^{2}(U-\xi V)-f^{\prime}\left(1+\xi^{2}\right)+4 f \xi$,

and

$\frac{\mathrm{d} w}{\mathrm{~d} \xi}=-2 V f^{2}+6 f$

Multiply both sides of Eq. (25a) with the term $\frac{1-3 \xi^{2}}{\left(1+\xi^{2}\right)^{3}}$ (i.e., which is equal to $\frac{d}{d \xi}\left[\frac{\xi}{\left(1+\xi^{2}\right)^{2}}\right]$ ) and both sides of Eq. (25b) with the term $\frac{\xi}{\left(1+\xi^{2}\right)^{2}}$, respectively and using (18a) and (18b) we obtain

$\frac{\mathrm{d}}{\mathrm{d} \xi}\left[\frac{w \xi-f\left(3 \xi^{2}-1\right)}{\left(1+\xi^{2}\right)^{2}}\right]=0$.

which automatically gives 
$\frac{w \xi-f\left(3 \xi^{2}-1\right)}{\left(1+\xi^{2}\right)^{2}}=\lambda$

(26a)

where $\lambda$ is a constant of integration, which is related to the flux parameter to be determined hereafter. Introduction of (26a), (17a) and (17b) into (25a) we obtain

$\frac{\mathrm{d} f}{\mathrm{~d} \xi}=\frac{4 \xi}{\left(1+\xi^{2}\right)^{3}} f^{2}+\frac{1}{\xi} f-\frac{1+\xi^{2}}{\xi} \lambda$

In order to solve (27), we apply the Riccati transformation [7]

$f=-\frac{\left(1+\xi^{2}\right)^{3}}{4 \xi} \frac{1}{u} \frac{\mathrm{d} u}{\mathrm{~d} \xi}$

where $u(\xi)$ is yet an unknown function of $\xi$. Substituting (28) in (27) we obtain the following linear ODE

$\frac{d^{2} u}{d^{2} \xi}+2 \frac{2 \xi^{2}-1}{\xi\left(1+\xi^{2}\right)} \frac{d u}{d \xi}-\frac{4 \lambda}{\left(1+\xi^{2}\right)^{2}} u=0$.

A new variable $z(\zeta)$ is defined now as follows

$z=\frac{1}{1+\xi^{2}}$.

Substituting (30) in (29) then yields the hypergeometric equation

$z(1-z) \frac{d^{2} u}{d z^{2}}+\left(z-\frac{1}{2}\right) \frac{d u}{d z}-\lambda u=0$,

with the general solution

$u(z)=C_{1}\left(C F_{1}(z)+z^{3 / 2} F_{2}(z)\right)$, $\frac{\mathrm{d} u}{\mathrm{~d} z}=C_{1}\left(-C 2 \lambda F_{3}(z)+\frac{3}{2} \sqrt{z} F_{2}(z)+\frac{2}{5}\left(\lambda-\frac{3}{4}\right) z^{3 / 2} F_{4}(z)\right)$,

where the hypergeometric functions $F_{3}(z)$ and $F_{4}(z)$ are given by

$F_{3}(z)=F\left(a,-a, \frac{1}{2} ; z\right)$

$F_{4}(z)=F\left(\frac{3}{2}+a, \frac{3}{2}-a, \frac{7}{2} ; z\right)$.

Using the website https://www.wolframalpha.com/, we obtain simple forms to the above hypergeometric functions

$F_{1}(z)=(1-2 z) \cos \theta+2 a \sqrt{1-z} \sqrt{z} \sin \theta$,

$F_{2}(z)=\frac{3}{4 \lambda}\left(\frac{(2 z-1) \sin \theta}{2 a z^{3 / 2}}+\frac{\sqrt{1-z} \cos \theta}{z}\right)$

$F_{3}(z)=\cos \theta$,

and

$F_{4}(z)=\frac{15}{4 \lambda(4 \lambda-3)}\left(\frac{((6-4 \lambda) z-3) \sin \theta}{2 a z^{5 / 2}}+\frac{3 \sqrt{1-z} \cos \theta}{z^{2}}\right)$,

where

$\theta=2 a \sin ^{-1} \sqrt{z}$.

Introducing (30) into (28) we obtain

$f(z)=-\frac{1}{2 z} \frac{1}{u} \frac{\mathrm{d} u}{\mathrm{~d} z}$.

Substituting (32) and (33) into (35) we obtain

$f(z)=\frac{1}{2 z} \frac{\frac{3}{4 a} \tan \theta-2 C \lambda}{\left(2 a C \sqrt{z(1-z)}+\frac{3}{8 \lambda a}(2 z-1)\right) \tan \theta-C(2 z-1)+\frac{3}{4 \lambda} \sqrt{z(1-z)}}$.

We simplify (36) by introducing (30) into (34e) to obtain

where

$F_{1}(z)=F\left(-1+a,-1-a,-\frac{1}{2}, z\right)$,

$F_{2}(z)=F\left(\frac{1}{2}+a, \frac{1}{2}-a, \frac{5}{2}, z\right)$,

are expressed via hypergeometric functions [1], $C$ and $C_{1}$ are constants to be determined below and $a=\sqrt{1-\lambda}$. Using the properties of the hypergeometric series, we obtain from (32), (32a) and (32b) the expression for $\frac{d u(z)}{d z}$

$$
f(\xi)=\frac{\lambda\left(1+\xi^{2}\right)^{2}}{1-\xi^{2}-2 \sqrt{1-\lambda} \xi \tan \left(2 \sqrt{1-\lambda} \cot ^{-1} \xi+\tan ^{-1} \frac{3}{8 C \lambda \sqrt{1-\lambda}}\right)} .
$$

In order to determine $C$ we use the following property of Eq. (27)

$f^{\prime}(\xi)=-f^{\prime}(-\xi)$. 
Integration of (38a) gives the following symmetrical property of (37), with respect to $\xi=0$, as follows

$f(\xi)=f(-\xi)$.

According to (38b), the denominator of the solution for $f(\xi)$ in (37) must be equal to the denominator of the solution for $f(-\xi)$ as follows

$$
\begin{aligned}
& \tan \left(2 \sqrt{1-\lambda} \cot ^{-1} \xi+\tan ^{-1} \frac{3}{8 C \lambda \sqrt{1-\lambda}}\right) \\
& +\tan \left(2 \sqrt{1-\lambda} \cot ^{-1}(-\xi)+\tan ^{-1} \frac{3}{8 C \lambda \sqrt{1-\lambda}}\right)=0
\end{aligned}
$$

which automatically yields $C \rightarrow \infty$. As a result, (37) reduces to

$$
f(\xi)=\frac{\lambda\left(1+\xi^{2}\right)^{2}}{1-\xi^{2}+2 \sqrt{\lambda-1} \xi \tanh \left(2 \sqrt{\lambda-1} \cot ^{-1} \xi\right)},
$$

which is valid in the domain $-\xi_{b} \leq \xi \leq+\xi_{b}$, as will be explained later. Using the results of (38b), together with (16a) we obtain the following symmetrical property (with respect to the axis $\hat{x}=0$ and $\hat{y}=0$ ) of the saturation profile

$S(\hat{x}, \hat{y})=S(-\hat{x}, \hat{y})=S(\hat{x},-\hat{y})=S(-\hat{x},-\hat{y})$.

\section{Some more properties of the similarity function $f(\xi)$}

By equation $\xi$ to zero, we obtain from (32)

$f(0)=\lambda$.

This mean that the parameter $\lambda$ must be greater than zero (i.e., $\lambda>0$ ) to assure that the oil saturation $S(\hat{x}, \hat{y})$, given in (16a), is positive. In addition to this we obtain from (27) (and from (38a) as well)

$f^{\prime}(0)=0$.

The property of $f(\xi)$ at infinity can be obtain from (40) as follow

$f \sim-\lambda \xi^{2}, \quad|\xi| \rightarrow \infty$.

The negative value for $f(\zeta)$ shows that the similarity solution $f(\zeta)$ is not uniformly valid everywhere since there is no physical meaning to a negative value for $f(\xi)$. However, the result (43) shows that there is a boundary parameter $\xi_{b}$ which divided between the region that possesses "physical meaning" exists in the range $-\xi_{b}<\xi<+\xi_{b}$ and the "no physical meaning" region lying at $\left|\xi_{b}\right|<\xi<|\infty|$ which is part of the "free layer" zone. Hence, the boundary parameter $\xi_{b}$, is the point at which the function $f(\xi)$ passes from a positive value to a negative value and possesses the following property

$f\left(\left|\xi_{b}\right|\right) \rightarrow|\infty|$.

This parameter can be determined by equating the denominator of (40) to zero

$1-\xi_{b}^{2}+2 \sqrt{\lambda-1} \xi_{b} \tanh \left(2 \sqrt{\lambda-1} \cot ^{-1} \xi_{b}\right)=0$.

Figure 2 shows the dependence of the flux parameter $\lambda$ on $\xi_{b}$. It can be observed that as $\lambda$ becomes large, the boundary parameter $\xi_{b}$ becomes large as well with the following dependence

$\xi_{b} \approx 1.66 \times \sqrt{\lambda}, \quad \lambda \rightarrow \infty$.

\section{Calculation of the oil fluxes on $\hat{x}=0$}

In this section we will investigate the behavior of saturation flux on $\hat{x}=0$, where the injection wells are located. To this end we express the saturation flux in terms of the self-similar variable. Accordingly, we substitute (18a) and (18b) in (20a) and (20b) and obtain expressions for the flux components

$g=\frac{-2 \xi^{3}+6 \xi}{\left(1+\xi^{2}\right)^{3}} f^{2}-f^{\prime}$

and

$h=\frac{2-6 \xi^{2}}{\left(1+\xi^{2}\right)^{3}} f^{2}-2 f+f^{\prime} \xi$.

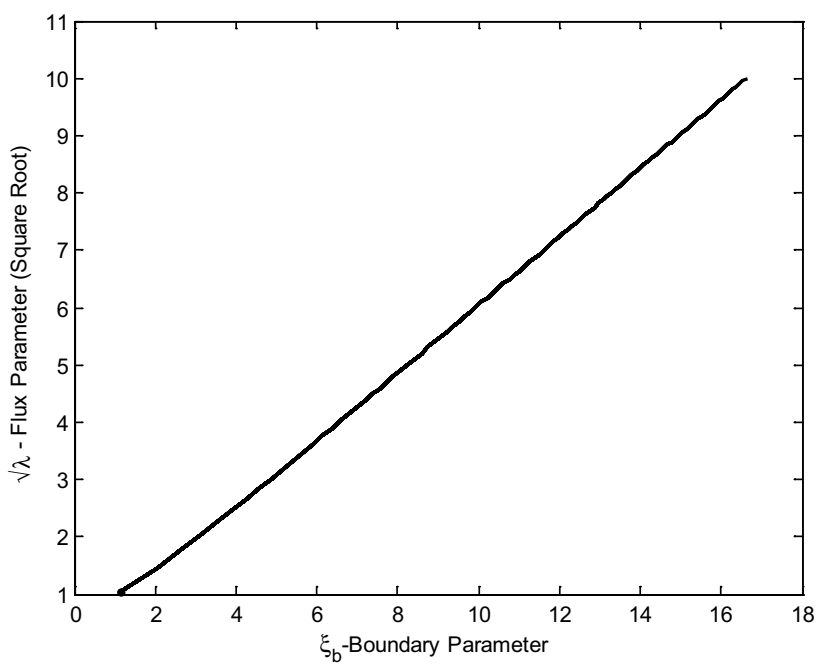

Fig. 2 Dependence of the flux parameter $\sqrt{\lambda}$ on $\xi_{b}$ 
The behavior of the flux components (20a) and (20b) at $\xi=0$ (i.e., $\hat{x}=0$ ) are obtained by the introduction of (42a) and (42b) into (47a) and (47b)

$g(0)=0$,

and

$h(0)=2 \lambda^{2}-2 \lambda$.

Substituting (48a) and (48b) into (19a) and (19b) we obtain the fluxes values on the $\hat{x}$ axis

$J_{\hat{x}}(0, \hat{y})=0$,

and

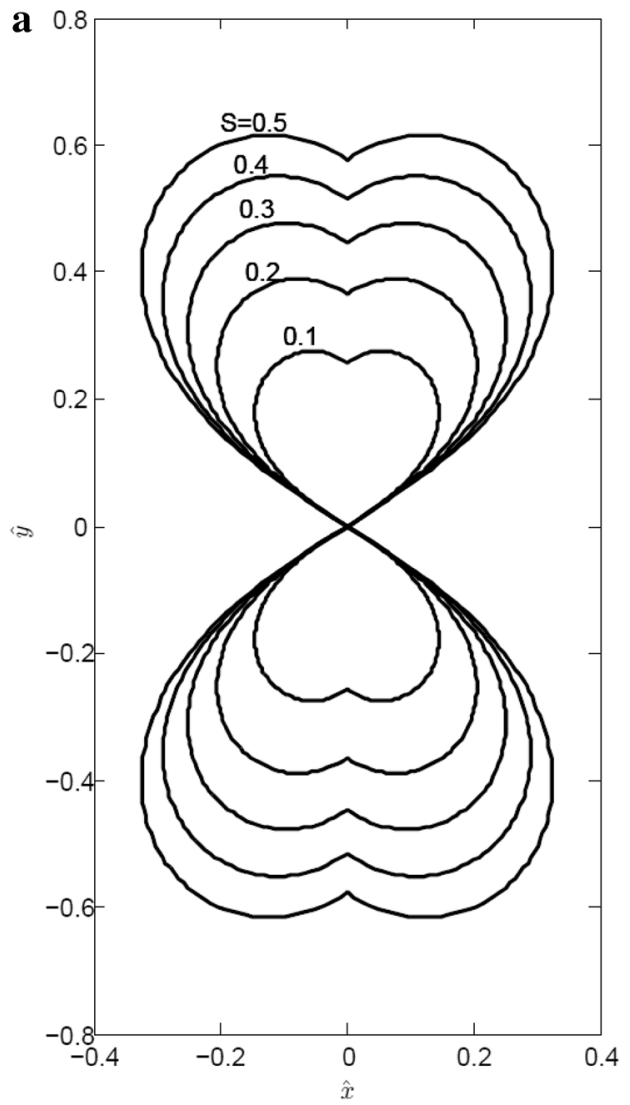

$J_{\hat{y}}(0, \hat{y})=2 \lambda(\lambda-1) \hat{y}$.

The result (49a) indicates that the $\hat{y}$ axis behaves as an impermeable barrier for the oil saturation flux in the $\hat{x}$ direction. In addition to this, the result (49b) indicates that for $\lambda>1$, the oil saturation flux possesses two branches on the $\hat{y}$ axis. The first one is directed from $\hat{y}=0$ to the $+\hat{y}$ direction and the other branch is directed from $\hat{y}=0$ to the $-\hat{y}$ direction, namely the upstream directions, as was previously defined.
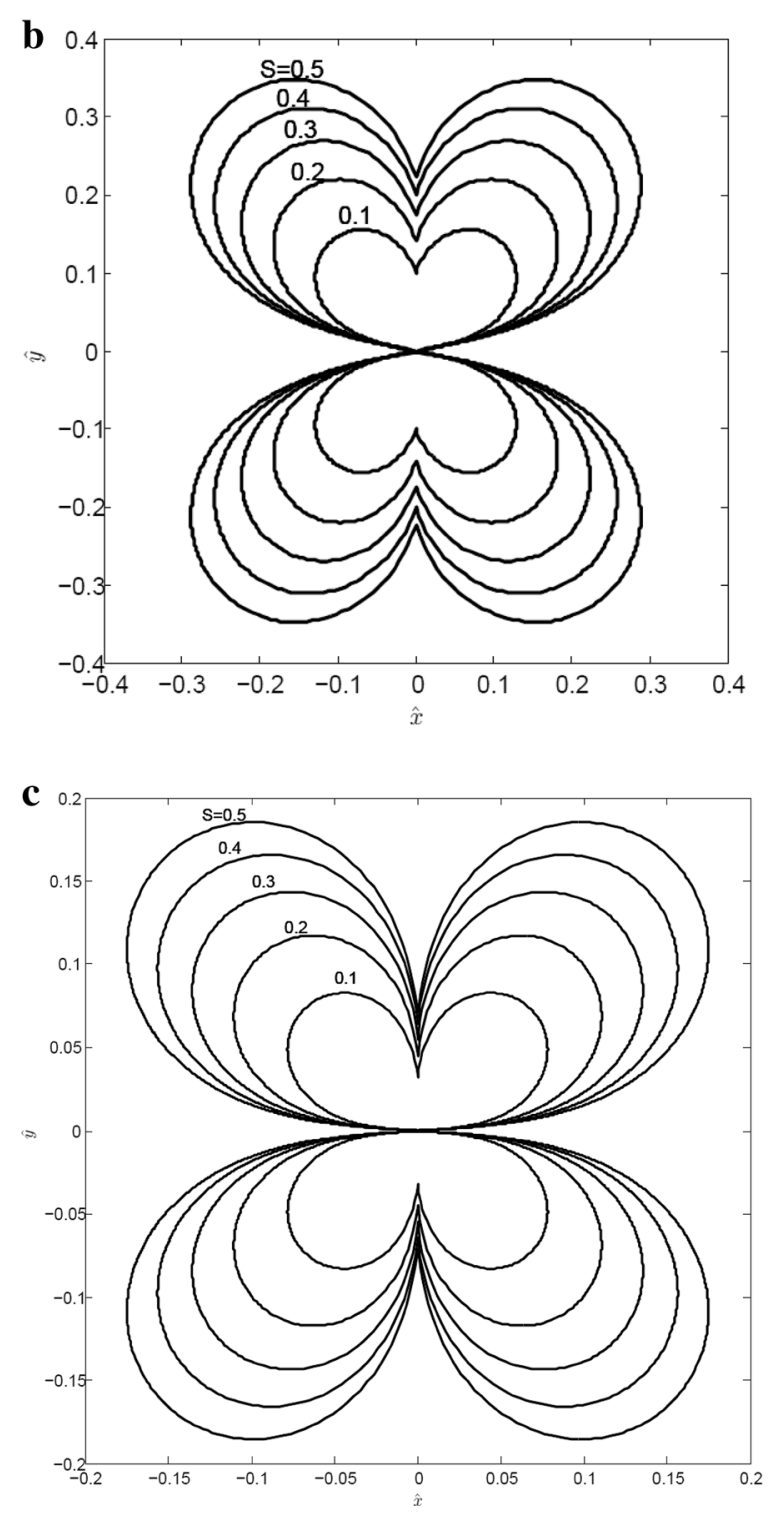

Fig. 3 Distribution of oil iso-saturation path lines around two extraction and two injection (2E/2I) wells located in the origin a $(m=n=2$ and $\lambda=1.5), \mathbf{b},(m=n=2$ and $\lambda=10), \mathbf{c}(m=n=2$ and $\lambda=100)$ 


\section{Results and discussion}

Figures $3 a-c$ depict the distribution of the oil saturation for three values of the oil flux parameter $\lambda$ [i.e., $m=n=2$ for all figures and hence $\tilde{S}=S$, see (12)]. It can be observed that as the oil flux parameter $\lambda$ becomes larger, the dimension of the saturation zone is reduced. On the contrary, when the value of the oil flux is low, smaller amounts of oil are transported by relatively large amounts of water from the injection wells toward the extraction wells, resulting in a larger transition zone. That is to say, the convection mechanism takes place over the dispersion process as the flux parameter becomes higher. As such, iso-saturation paths tend to possess relatively steep gradients which assure the domination of the convection over the dispersion mechanism (e.g., the arc over the area of oil-water menisci increases and the oil convective transport acts as a significant oil transport mechanism, comparable to the capillary dispersion.)

We now illustrate the applicability of the obtained solution by considering four wells ( $2 \mathrm{E} / 2 \mathrm{l}$ ) that are arranged in a quadrupole configuration in an aquifer, heavily polluted with oil. Let us assume that two hypothetical observation wells are lying at two known distances from the origin $(x=0, y=0)$. Such saturation data can be obtained by using elevation measurements of the water and the oil from the observation well [8]. Based on such data, we will illustrate how the actual position of the iso-saturation lines in the $x-y$ domain can be determined. Toward this end, we use the flowing hypothetical data as follows
(a) $S_{1}=0.1$ (at $x_{1}=0 \mathrm{~m}, y_{1}=5 \mathrm{~m}$ ),
(b) $S_{2}=0.3$ (at $x_{2}=10.0 \mathrm{~m}, y_{2}=10.0 \mathrm{~m}$ ),

Introducing the set of the data (a) into Eq. (16a) and (42a) we obtain

(c) $\frac{S_{1}}{y_{1}^{2}}=\frac{\lambda}{L^{2}}$

Combining (b), (c) together with (16b) and (40) we obtain the following implicit equation

(d) $\frac{S_{2}}{S_{1}} \times \frac{y_{1}^{2}}{y_{2}^{2}}=\frac{\left(1+\xi_{2}^{2}\right)^{2}}{1-\xi_{2}^{2}+2 \sqrt{\lambda-1} \xi_{2} \tanh \left(2 \sqrt{\lambda-1} \cot ^{-1} \xi_{2}\right)}$,

where

(e) $\xi_{2}=\frac{x_{2}}{y_{2}}$.

By using a trial and error technique we obtain

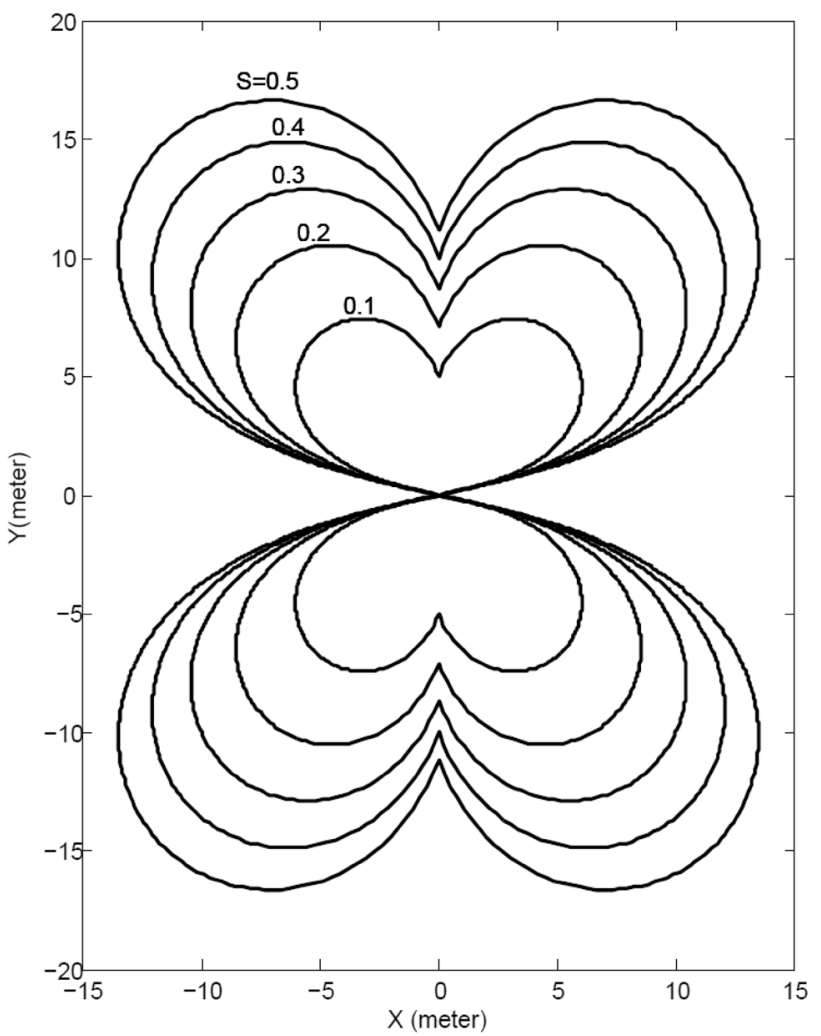

Fig. 4 Distribution of oil iso-saturation path lines around two extraction and two injection (2E/2l) wells located in the origin $(m=n=2$ and $\lambda \approx 8.115, L \approx 45 \mathrm{~m})$

Using (c) and (f) we obtain the characteristic length of the aquifer

(g) $L=y_{1} \sqrt{\frac{\lambda}{s_{1}}} \approx 45 \mathrm{~m}$.

No we will use the obtained parameter to calculate the actual position of the iso-saturation lines as can be observed in Fig. 4.

It should be noted that the solution for $\tilde{S}(\hat{x}, \hat{y})$ in the form (7a) does not generally reproduce the actual saturation but rather exhibits the following dependence

$\tilde{S}(\hat{x}, \hat{y}) \rightarrow \infty, \quad$ as $\hat{x}, \hat{y} \rightarrow \pm \infty$.

The existence of the self-similar functional form (16a) and (16b) thus reflects the tendency of the solution $\tilde{S}(\hat{x}, \hat{y})$ to "overlook" the actual saturation specified in large distance $\hat{y}$ while it eventually tends towards the similarity solution for small distances from the origin, in the transition zone. The free layer zone can be related to the zone where the similarity solution possesses no physical meaning (at $\left.\left|\xi_{b}\right|<\xi<|\infty|\right)$.

(f) $\lambda \approx 8.115$. 


\section{Summary and conclusions}

The steady-state distribution of oil contaminants in a saturated aquifer is analyzed and simulated by applying a geometrical self-similar model. The model employs the basic equations of the two-phase flow. In addition we assume some power law relationship which is supposed to exist between oil saturation, capillary pressure and relative permeability. The self-similar model pertains to cases wherein both space coordinates in the oil transport equations can be replaced by a single variable.

The influences of the extraction and the injection wells, located in quadrupole configuration and without regional flow, are lumped via the single oil flux parameter $\lambda$. It is found that decreasing the oil flux parameter led to the domination of the capillary dispersion processes compared with the convection process. This leads to an increasing of the transition zone dimensions.

It was shown that the obtained solution for $\tilde{S}(\hat{x}, \hat{y})$ in the form (16a) is complete and valid in the transition zone, which is enclosed by the iso saturation line $S=0.5$, defined in (16a) and (16b). The obtained solution does not generally reproduce the actual saturation beyond the transition zone and is blows up as $y$ become large. However, it eventually tends towards the similarity solution (16a) and (16b) in the transition zone where $S \leq 0.5$. In addition to this, it was proved that the size and the steady state oil saturation distribution in the transition zone is depends on the oil flux only and it is independent on the size of the free layer zone (which is unknown).

It was also shown how to use the model jointly with data collected from several observation wells to evaluate the extension of the oil in the transition zone and to check more comprehensive numerical treatments of the oil-water transport in aquifers.

\section{Compliance with ethical standards}

Conflict of interest The author declare that there is no conflict of interest.

\section{References}

1. Abramowitz M, Stegun IA (1970) Handbook of mathematical functions. Dover, New York

2. Anderson El, Mesa E (2006) The effects of vertical barrier walls on the hydraulic control of contaminated groundwater. Adv Water Resour 29(1):89-98

3. Antonacci T, Lee ES, Kim Y (2013) Characterizing and predicting contaminant transport in the Newport Wellfield aquifer, Ohio. Geosci J 17(4):465-477

4. Barenblatt GI (1979) Similarity, self-similarity and intermediate asymptotics. Consultants Bureau, New York

5. Bear JJ, Cheng HDA (2010) Modeling groundwater flow and contaminant transport, vol 23. Theory and applications of transport in porous media. Springer, Dordrecht

6. Campos LMBC (2010) Complex analysis with applications to flows and fields. CRC Press, Boca Raton

7. Kamke E (1959) Differentialgleichungen Losungsmethoden und Losungen. Chelsea Publishing Co., New York

8. Lenhard RJ, Parker JC (1990) Estimation of free hydrocarbon volume from fluid levels in monitoring wells. Ground Water 28(1):57-67

9. Mays DC, Neupauer RM (2010) Engineered well injection and extraction to enhance mixing in aquifers. In: Palmer RN (ed) World environmental and water resources congress 2010: challenges of change. American Society of Civil Engineers, Reston, pp 715-722

10. Payatakes AC (1982) Dynamics of oil ganglia during immiscible displacement in water wet porous media. Ann Rev Fluid Mech 14(1):365-393

11. Pistiner $A$ (2007) Oil plume distribution in an anisotropic porous medium. Transp Porous Media 70:293-304

12. Pistiner $A$ (2009) Oil plume distribution in an aquifer near an impermeable barrier. Transp Porous Media 76:67-75

13. Pistiner A, Shapiro M (1996) A model of groundwater pollution from an underground source. Water Resour Res 32(7):2311-2314

14. Pistiner A, Shapiro M, Rubin H (1990) Similarity solutions for immis-cible phase migration in porous media: an analysis of free bound-aries. Transp Porous Media 5:491-515

15. Ramakrishnan TS, Wilkinson D, Dias M (1988) Effect of capillary pressure on the approach to residual saturation. Transp Porous Media 3:51-79

16. Satkin RL, Bedient PB (1988) Effectiveness of various aquifer restoration schemes under variable hydrogeologic conditions. Groundwater 26(4):488-498

17. Zeidan BA (2017) Groundwater degradation and remediation in the Nile Delta Aquifer. In: Negm AM (ed) The Nile Delta, Handbook of environmental chemistry. Springer International Publishing AG, Basel, pp 159-232

Publisher's Note Springer Nature remains neutral with regard to jurisdictional claims in published maps and institutional affiliations. 